\title{
Elemente einer Soziolinguistik Nordafrikas ${ }^{1}$
}

\author{
H. Ekkehard Wolff \\ Institut für Afrikanistik, Universität Leipzig, Beethovenstrasse 15, D-04107 Leipzig \\ E-mail: ekkehard.wolff@gmx.de
}

\begin{abstract}
The sociolinguistics of the Northern African states is relatively obscure in terms of international research, since these regions do not feature significantly in the areas of Arabic studies/Orientalism, Romance studies or classical African studies. A similar problem holds for the scientific classification of studies on Berber. This article develops an africanist-sociolinguistic approach to central questions refering to multilingualism in the context of historically unfolding geo- and sociolinguistic profiles of Northern African nations. This will be discussed within the framework of a historical linguistic stratigraphy. The article broadens the concept of diglossia as used in the arabophonic context in order to include a multilingual dimension (polyglotism), and discusses models of conflict avoidance between native speakers of Arabic, Berber and French, respectively. The article focuses on situationally adequate language political arrangements, especially in the area of education, on the basis of L1-based additive bi- and trilingualism models.
\end{abstract}

Keywords: African studies, Arabic, Berber, education, diglossia, French, Maghreb, multilingualism, polyglotism, sociolinguistics, language policy

Schlüsselbegriffe: Afrikanistik, Arabisch, Berber, Bildung, Diglossie, Französisch, Maghreb, Mehrsprachigkeit, Polyglossie, Soziolinguistik, Sprachenpolitik

\section{Einleitung: Nordafrika - eine Region ,zwischen den Disziplinen“}

In der europäischen Wissenschaftsorganisation befindet sich die soziolinguistische Forschung mit Bezug auf die Staaten Marokko, Algerien, Tunesien, Libyen und Ägypten in einer Situation quasi zwischen allen Stühlen. Einerseits erklärt sich bezüglich der Sprachen Arabisch und Berberisch traditionell die sog. Orientalistik im Sinn der Wissenschaft von Geschichte, Sprachen und Kulturen des Vorderen Orients für fachlich zuständig, deren klassische Arbeitsteilung zwischen Arabistik, Islamkunde und Semitistik sowie vor allem die traditionelle regionale Fokussierung auf Gebiete außerhalb Afrikas nun aber wiederum einer intensiven Beschäftigung mit der Soziolinguistik der Region Nordafrika entgegensteht. Als Zweites kommt angesichts der bedeutenden Rolle des Französischen in Nordafrika, die der kolonialpolitischen Vergangenheit und modernen Globalisierungsaspekten geschuldet ist, die in der Romanistik angesiedelte Frankophonieforschung ins Spiel. Als Drittes wären partikuläre Forschungsinteressen aus einer allgemeinen Soziolinguistik zu berücksichtigen, für die der arabophone Raum generell als Lehrbuchfall für „Diglossie“ (s. weiter unten) interessant ist. Geographisch gehören diese Länder wiederum und eigentlich in das 
Arbeitsgebiet der klassischen Afrikanistik, die als solche allerdings auf deutsche und einige europäische Lehr- und Forschungseinrichtungen beschränkt ist und in deren geographischem Fokus zudem zumeist das sog. subsaharische Afrika liegt; man vgl. auch das jüngere angloamerikanische Konzept der „African Studies“, das sich praktisch ausschließlich mit dem sog. subsaharischen Afrika beschäftigt. Insofern liegt Nordafrika auch weitgehend im Schatten des Interesses der internationalen African Studies wie der deutschsprachigen Afrikanistik - sehr zu Unrecht, wie in diesem Beitrag dargelegt werden soll.

In den zuständigen Philologien der Arabistik, Berberologie und Französistik hat das komplexe, durch Multilingualismus und Polyglossie gekennzeichnete soziolinguistische Profil der Maghrebstaaten keine echte Forschungsheimat gefunden. Die Arabistik beschäftigt sich primär mit Literatur und medialer Verwendung des (Modernen Hoch-)Arabischen sowie dem Klassisch-Arabischen der qur'anischen Tradition. Die als sog. „vulgärarabische Dialekte“ bezeichneten Volkssprachen der alltäglichen Kommunikation, die von den Sprechern inzwischen terminologisch quasi nationalisiert wurden und als Ägyptisch, Algerisch, Marokkanisch, Tunesisch etc. bezeichnet werden, kommen im normalen akademischen Betrieb kaum vor und werden von der traditionellen Arabistik marginalisiert wenn nicht gar stigmatisiert. Die Berberologie, die im traditionellen Wissenschaftsbetrieb unglücklich zwischen Orientalistik und Afrikanistik angesiedelt ist und dadurch keiner der beiden Fachdisziplinen so recht am Herzen zu liegen scheint, beschäftigt sich primär mit deskriptiver Dialektologie und den notwendigen Erstanalysen von Berbervarietäten/-sprachen, ggf. auch mit der weitgehend noch als strittig behandelten Frage, ob es sich beim Berberischen um mehrere (und welche) Einzelsprachen handelt, oder ob nicht alle Varietäten des Berberischen als mundartliche Realisierungen einer einzigen Sprache anzusehen seien, man vgl. die traditionellen französischen Begrifflichkeiten von „la langue berbère“ vs. „parlers berbères“. Die Frankoromanistik wiederum beschäftigt sich bzgl. Nordafrika allenfalls mit varietätenlinguistischen Analysen des Französischen nordafrikanischer Sprecher.

Dem gegenüber steht der umfassendere afrikanistische Ansatz, wie er seit Beginn des 20. Jahrhunderts an den Universitäten Berlin, Leipzig und Hamburg, aber auch Wien, eine eigenständige neue Wissenschaftsdisziplin begründet hat. Diesem Anspruch entspricht zunächst die gesamtkontinentale Perspektive auf Afrika, die sich schon aus der fundamentalen linguistischen Situation ergibt: So gehören Ägypten ebenso wie der Maghreb sprachlich zum Verbreitungsgebiet der Afroasiatischen Sprachen, das weit über den Südrand der Sahara hinaus bis in die Sahelzone Westafrikas und bis weit über das äthiopische Hochland hinaus nach Süden bis Tansania reicht. ${ }^{2}$ Entsprechend sehen sich viele deutschsprachige Afrikanisten in der wissenschaftlichen Tradition, nach der die Großregion Nordafrika, insbesondere hinsichtlich des Berberischen, quasi natürliches Forschungsgebiet der Afrikanistik ist. Ein weiteres Merkmal des afrikanistischen Ansatzes ist es, sich einerseits bezüglich Modellbildung an allgemeinen (sozio-)linguistischen Theorien $\mathrm{zu}$ orientieren, und andererseits alle involvierten Sprachen und Varietäten, d.h. Arabisch, Berberisch und Französisch unter den Bedingungen ihrer Nachbarschaft und Rivalität ins Auge zu fassen versteht sich diese Afrikanistik doch nicht nur als die Wissenschaft von den afrikanischen Sprachen, sondern auch als die Wissenschaft von „Sprache in Afrika“, und dies schließt die nach Afrika importierten oder dort eigene Varietäten ausprägenden nicht-afrikanischen Sprachen mit ein. Die Afrikanistik tut dies in Nordafrika, wie es ihrer Herangehensweise auch in anderen Regionen Afrikas entspricht, nämlich unter Einbeziehung aller relevanten regionalspezifischen historischen und demographischen Gegebenheiten, besonders auch hinsichtlich 
historischer Vergleiche der prä- und postkolonialen Situationen sowie unter dem Aspekt des typologischen Vergleichs mit anderen Großregionen des afrikanischen Kontinents.

Die Afrikanistische Soziolinguistik stellt damit Modelle, Begrifflichkeiten und Typologien zur Verfügung, die es erlauben, sich unter Erhalt der Vergleichbarkeit mit anderen afrikanischen Großregionen intensiv mit der Soziolinguistik Nordafrikas auseinander zu setzen. Hierzu gehören die Typologie von „Mehrsprachigkeitsprofilen“, das Ausmaß und die Ausprägung von individuellem und institutionellem Multilingualismus, Polyglossie-Reflexe in Sprachenrecht und Sprachenpolitik, attitudinale Divergenzen, pädagogische und didaktische Strategien beim Erst-, Zweit- und Fremdsprachenerwerb im Bildungssektor, sowie das Potenzial der Sprachen als Ressourcen für nachhaltige soziokulturelle, politische und ökonomische Entwicklung und gesellschaftliche Modernisierung. Wir werden im Folgenden auf diese Aspekte unter dem räumlichen Bezug auf Nordafrika eingehen.

\section{Zum Begriff der Mehrsprachigkeit in Afrika allgemein, und in Nordafrika im besonderen}

Mehrsprachigkeit (Multilingualismus) ist nur scheinbar ein trivialer Begriff, der als soziolinguistischer Terminus zur Identifizierung von sehr verschiedenen Phänomenen führt. Multilingualismus im engeren Sinn umfasst zum einen den individuellen Multilingualismus von Sprechern, deren linguistische Biographie durch simultanen oder sukzessiven Erwerb von zwei oder mehr Sprachen geprägt ist. Dabei kann es sich um frühkindlichen, d.h. vorschulischen Zweit- und Drittsprachenerwerb handeln, der spontan und ungesteuert in der Familie oder in der peer-group quasi spielend auf der Straße geschieht. Oder es handelt sich um gesteuerten Spracherwerb durch das System der formalen Bildung, der also in charakteristischer Weise in der Schule oder bereits in der Vorschule geschieht, sei es abrupt gleich bei der Einschulung, sei es gleitend im Verlaufe der Schulzeit. Dieser individuelle Mehrsprachigkeitserwerb war lange Zeit Domäne der Psycholinguistik bzw. Psychosoziolinguistik und wurde von der Afrikanistik bis in die jüngere Vergangenheit kaum als Forschungsobjekt wahrgenommen. Auch die moderne Bilingualismusforschung im Rahmen einer umfassenderen Angewandten Linguistik hat es schwer, in einer sich überwiegend „regionalwissenschaftlich“ verstehenden Afrikanistik Fuß zu fassen. Studien zum frühkindlichen Mehrsprachigkeitserwerb in Afrika sind daher rar. Darüber hinaus würde das Phänomen der sog. Halbsprachigkeit (Semilingualismus) eine besondere Beachtung verdienen, d.h. der nur unvollkommenen Beherrschung von Zweit- und Drittsprachen, insbesondere wenn es sich um die Amts- und Bildungssprachen der jeweiligen Staaten handelt. Bildungserfolg und Integrationsfähigkeit in einer modernen Gesellschaft hängen in entscheidendem Maße von einer möglichst vollkommenen Beherrschung der jeweiligen Amts- und Bildungssprache ab - in Afrika ebenso wie in Europa bzw. Deutschland (hier vor allem für Menschen mit „Migrationshintergrund“). Entsprechende Sprachstandsuntersuchungen in Afrika sind, wenn überhaupt, durchaus selten; die Existenz und das Ausmaß von Semilingualismus als der nachhaltigen soziokulturellen „Modernisierung“ der Gesellschaften abträgliches Phänomen wird, zumal was den formalen Bildungsbereich betrifft, entweder als Problem nicht gesehen oder totgeschwiegen. ${ }^{3}$

Dem gegenüber steht institutioneller Multilingualismus, eines der klassischen Themen der afrikanistischen Soziolinguistik, bei dem es sich einerseits um ungeplante, d.h. spontan gewachsene, andererseits um politikgesteuerte, d.h. geplante Mehrsprachigkeit handeln kann, 
die sich zudem von Fall zu Fall auf verschiedenen Ebenen manifestiert. Da ist zum einen die lokale Ebene, z.B. hinsichtlich lokaler Primarschulen in multilingualen Kontexten mit der Frage, welche der Erst- und/oder Zweitsprachen der Kinder als Unterrichtsmedien überhaupt verwendet werden. Ein anderes Beispiel betrifft die informelle Institutionalisierung von sog. Marktsprachen zur Überwindung von Sprachbarrieren zwischen Marktbenutzern, die von Hause aus unterschiedliche Erst- bzw. Muttersprachen sprechen. Auf regionaler Ebene prägen regionale linguae francae (auch als Verkehrs-, Handels- oder Arbeitssprachen apostrophiert) das Bild, deren Verbreitung mit dem Gegensatz zwischen ländlichen und städtischen Räumen korrelieren kann: Ländliche Räume sind eher durch ein höheres Maß an Monolingualität, urbane Zentren hingegen eher durch Multilingualität gekennzeichnet. Der Kontakt mit Behörden in einer Provinzhauptstadt bedeutet für viele Bewohner ländlicher Gebiete oft die Notwendigkeit, in einer zweiten oder dritten Sprache kommunizieren zu müssen. Die nationale Ebene der institutionellen Mehrsprachigkeit betrifft das juristisch-administrative und in der Praxis nicht immer geklärte Verhältnis von sog. Amts- und sog. Nationalsprachen (official languages vs. national languages), denen per Verfassung oder Dekret, also wiederum politikgesteuert bzw. de jure, oder auch nur durch faktischen Sprachgebrauch, also informell bzw. de facto, verschiedene und teilweise überlappende Domänen zugewiesen sind. Etliche afrikanische Staaten bekennen sich zu mehreren Amtssprachen, in den meisten Fällen handelt es sich um zwei oder drei Sprachen; das Maximum gilt für Südafrika mit seinen 11 in der Verfassung festgelegten Amtssprachen. In Fällen „offizieller“ institutioneller Mehrsprachigkeit mit Verfassungsrang finden wir in Afrika vielfach Kombinationen von einer indigenen und einer exogenen Sprache; allein Kamerun leistet sich, aufgrund einer besonderen kolonialpolitischen Ausgangssituation, den Luxus von zwei exogenen Amtssprachen (Französisch und Englisch).

Ein interessantes und relativ rezentes Phänomen individueller und gruppenspezifischer Mehrsprachigkeit betrifft die in den urbanen Agglomerationen der afrikanischen Megastädte entstehenden „Kiezsprachen“ (new urban vernaculars), wie z. B. das auf der Basis des Kiswahili entstandene Sheng in Nairobi und anderen kenianischen Städten, das auf der Basis des westafrikanischen Französischen entstandene Nouchi in Abidjan und anderen Städten der Côte d'Ivoire, das auf der Basis des Afrikaans entstandene Tsotsitaal und das Zulu-basierte Iscamtho/Isicamtho in Johannesburg und anderen Großstädten Südafrikas. Bei diesen Kiezsprachen, die ihren Ursprung in den subkulturellen städtischen Ghettos der Jugendbanden und Kleinkriminellen haben, handelt es sich um eine mehrschichtige Reaktion auf die herrschenden Verhältnisse: zum einen auf die als Kulturimperialismus wahrgenommenen Perpetuierung post- bzw. neokolonialer Abhängigkeiten, die sich im offiziellen Gebrauch der Sprachen der ehemaligen Kolonialmächte manifestiert, zum anderen als Ausdruck eines damit zusammenhängenden Generationenkonflikts sowie um den kulturellen Gegensatz zwischen ländlichen und städtischen Verhaltensmustern. Die offiziellen Sprachvarianten, ob nun des Französischen oder des Zulu, werden als ,uncool“ bewusst abgelehnt und linguistisch kreativ bis zur Unkenntlichkeit verballhornt und verfremdet, bis eine neue, das städtisch-afrikanische Lebensgefühl der jüngeren Generation symbolisierende, Sprachvarietät entsteht, für die keine der überkommenen Regeln und Normen mehr gelten (so wird etwa im Nouchi von Abidjan das normativ "korrekte“ hexagonale Französisch des ex-kolonialen Mutterlandes und der formalen Bildung als bagarre de Molière verspottet). Im Wesentlichen geht es hier auch um „Eigentum“ (ownership) an der Sprache: Wem „gehört“ das Englische in Kenia, das Französische in Kamerun, das Portugiesische in Angola etc.? Ist z.B. Englisch bereits eine „kenianische Sprache“ geworden, wie mancher kenianische Intellektuelle bereit ist zu 
behaupten? Wem ,gehört“ das West African Pidgin in Nigeria und Kamerun? Strategien, die ex-Kolonialsprachen zu ,,afrikanisieren“ - im Einzelfall also z.B. zu „kamerunisieren“ -, werden in Afrika durchaus ernsthaft als Strategien einer als überfällig empfundenen mentalen und linguistischen Dekolonisierung diskutiert. ${ }^{4}$

Multilingualismus im weiteren Sinn umfasst in Afrika aber auch ein Phänomen, das Whiteley (1974) für die kenianische Situation beschrieben und mit dem Begriff des „MultiMonolingualismus" verbunden hat. Gemeint ist hier das für große Teile Afrikas immer noch geltende Nebeneinander von monolingualen Regionen in einem politisch definierten Territorium, d.h. die Existenz vieler Sprachen auf dem nationalen Territorium, bei gleichzeitig hohen Raten von Monolingualismus bei der jeweiligen Bevölkerung (,territoriale Mehrsprachigkeit").

Dies sind einige der in Afrika weit verbreiteten Facetten von Mehrsprachigkeit, die auch bei einer Typologie der soziolinguistischen Situationen und Profile in Nordafrika zu berücksichtigen sein werden.

\section{Die geo- und soziolinguistischen Profile des nördlichen Afrikas}

Um ein umfassendes Bild der soziolinguistischen Situation des nördlichen Afrikas zu zeichnen, müssen wir auf eine Reihe von Faktoren blicken, die zu der spezifischen Komplexität dieser Situation beigetragen haben. Dies sind zum einen die sprachensoziologische Stratigraphie (territoriale Überlagerung mehrerer Sprachen) und deren historische Hintergründe, zum anderen die geolinguistische Distribution (regionale Sprachverbreitung), die gesellschaftlichen Domänen (Sprachfunktionen) sowie die Mitgliedschaft in internationalen politischen Organisationen auf der Basis gemeinsamer Sprachen. Wir werden diese Faktorenbündel im Folgenden etwas präziser einführen.

Hinsichtlich der sprachensoziologischen Stratigraphie und deren historischen Hintergründen unterscheiden wir drei linguistische Strata. Da ist zunächst ein prä-islamisch und vorarabisches Stratum 1, das durch eine Mehrzahl von Sprachen, die alle dem afroasiatischen Sprachstamm zugeordnet werden, geprägt war. Weiterhin können wir von einem arabischen Stratum 2 sprechen, das die frühe Islamisierung der Großregion widerspiegelt. Schließlich treffen wir auf ein kolonialzeitlich begründetes Stratum 3, das dem Französischen eine signifikante Rolle zukommen ließ.

Stratum 1 wird heute durch die aus prä-islamischer Zeit ererbten Berbersprachen repräsentiert, wie sie in mehr oder minder isolierten Sprachinseln in der Großregion zwischen Mauretanien bzw. Marokko im Westen und Ägypten im Osten, dem Mittelmeer im Norden und der zentralen Sahara im Süden verbreitet sind. Eine in Fachkreisen übliche Unterteilung der Berbersprachen in Gruppen von benachbarten Varietäten bietet sich aus dieser räumlichen Distribution an, man unterscheidet daher - nicht zuletzt in Ermangelung einer unstrittigen dialektologisch abgesicherten Untergliederung - eine Nordgruppe mit Tashelhit, Tamazight, Tarifit, Kabylisch, etc. in Marokko und Algerien, eine Ostgruppe mit Awjilah, Nefusi, Siwi, etc., eine Südgruppe in der südlichen Sahara mit Tamajeq Tamashek Tamahaq, den Varietäten der Sprache der Tuareg, sowie eine Westgruppe mit dem Zenaga unweit der mauretanischen Atlantikküste, so z.B. bei Hayward (2000:76f). Andere Autoren bezeichnen Siwi, Nefusi, die Sprache der Tuareg (Tamajaq o.ä.) und das Zenaga als Einzelsprachen an der 
Peripherie des Sprachgebietes und sprechen zusätzlich von zwei großen Dialektgruppen: Die Zenata-Dialekte (,Wüstenberberisch“) und das „Atlasberberische“" (so auch bei Wolff 1981b). Gelegentlich werden auch das Shluh oder Schilhische (Tashelhit), das Rif (Tarifit) sowie das Kabylische als eigenständige Berbersprachen bezeichnet. Die Bezeichnung Tamazight (,Sprache der Amazigh, d.h. der Freien“) gewinnt zunehmend an Bedeutung als übergeordnete Eigenbezeichnung für alle Berbersprachigen, gilt aber zunächst einmal als Bezeichnung für eine Untergruppe von Dialekten.

Aus historischer Sicht gehören in den Kreis der Berbersprachen vermutlich eine Reihe von heute ausgestorbenen Sprachen, die uns nur noch über Inschriften und/oder Fremdwörter und Namen in anderen, besser bekannten Sprachen überliefert sind; dazu gehören das Altlibysche/Numidische, das Alt-Mauretanische, das Guanche auf den Kanarischen Inseln, und möglicherweise das Alt-Iberische. Die Großregion Nordafrika wurde in historischer Zeit aber auch durch andere indigene Sprachen geprägt, die nicht zu den Berbersprachen gehören, aber dennoch weitläufig im Rahmen des afroasiatischen Sprachstammes mit diesen verwandt sind; sie sind heute ausgestorben und nur noch aus schriftlichen Sprachdenkmälern bekannt, wobei das Koptische als Liturgiesprache der koptischen Christen in Ägypten immer noch eine wichtige Funktion in der Gegenwart wahrnimmt. Die Rede ist vom Ägyptischen, das uns in verschiedenen Sprachstufen vom pharaonischen Altägyptisch der Hieroglyphen über das Mittelägyptische und Demotische bis zum Koptischen bekannt ist. ${ }^{5}$ Eine weitere wichtige Sprache in der Großregion in historischer Zeit war das Phönizische (Punisch), das der semitischen Sprachfamilie innerhalb des Afroasiatischen zugerechnet wird. Karte 1 zeigt die heutige Verbreitung des Berberischen sowie der mit ihm verwandten Sprachen des Afroasiatischen - ohne Berücksichtigung des über quasi die ganze Großregion Nordafrika verbreiteten Arabischen.

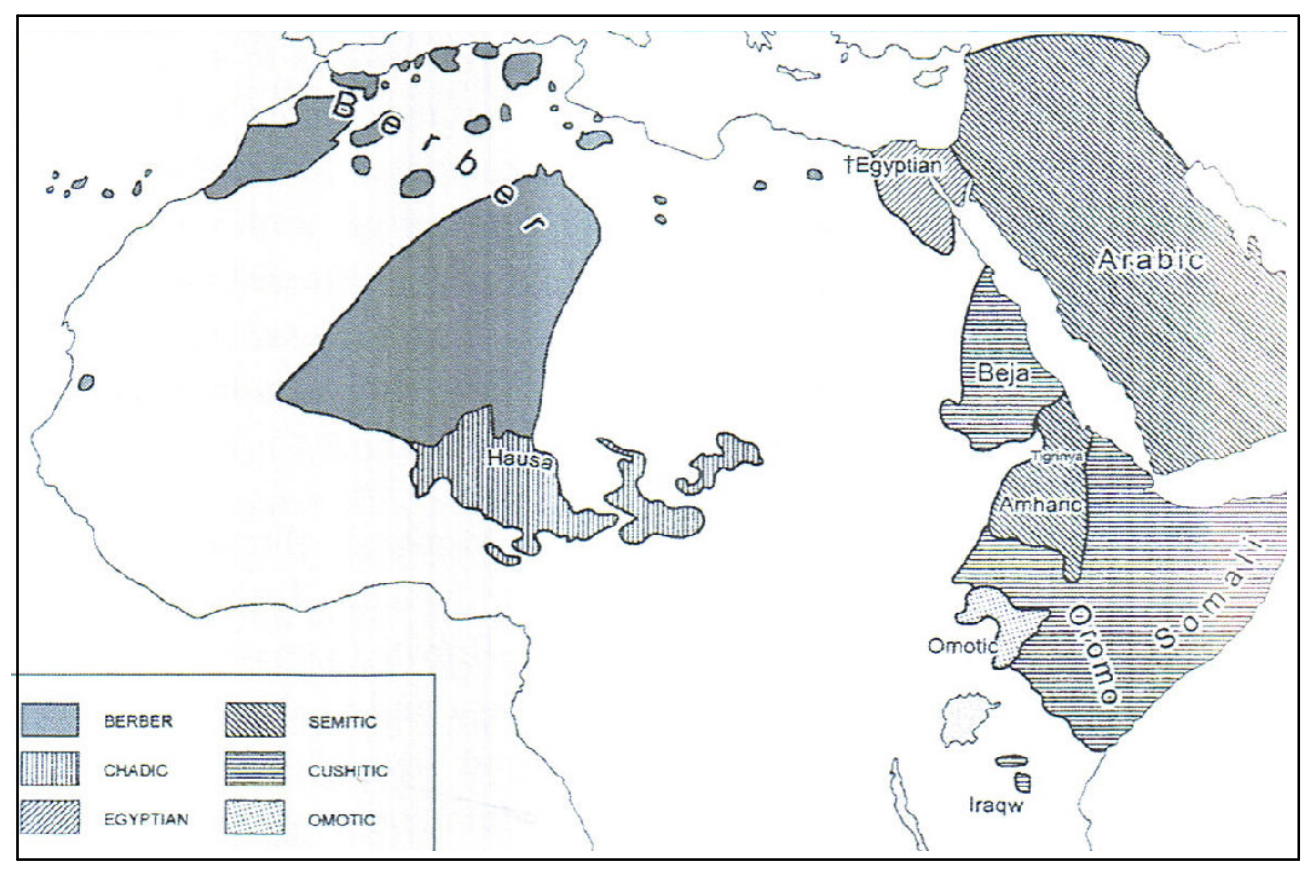

Karte 1. Die Verbreitung des Berberischen und seiner Afroasiatischen Schwesterfamilien (nach Heine/Nurse 2000:75) 
Stratum 2 geht auf die Islamisierung zurück, die im 7. und 8. Jahrhundert eine dynamische Ausbreitung über Nordafrika und von dort auf die Iberische Halbinsel erfuhr. Mit der Islamisierung einher geht die Überlagerung des ursprünglich vermutlich weitgehend geschlossenen berberophonen Sprachraumes; dies schafft ein über die Jahrhunderte stabiles Nebeneinander von arabischen und berberischen Varietäten, das mit einer robusten aber asymmetrischen Etablierung von Berberisch-Arabischem Bilingualismus auf Seiten der L1Berbersprachigen - aber nicht umgekehrt - einher gegangen sein dürfte. Karte 2 zeigt die Ausbreitung des Arabischen, einhergehend mit der Expansion des Islam, die bis zum 16. Jhd. weite Teile des subsaharischen Sahelgürtels erreicht hatte. ${ }^{6}$

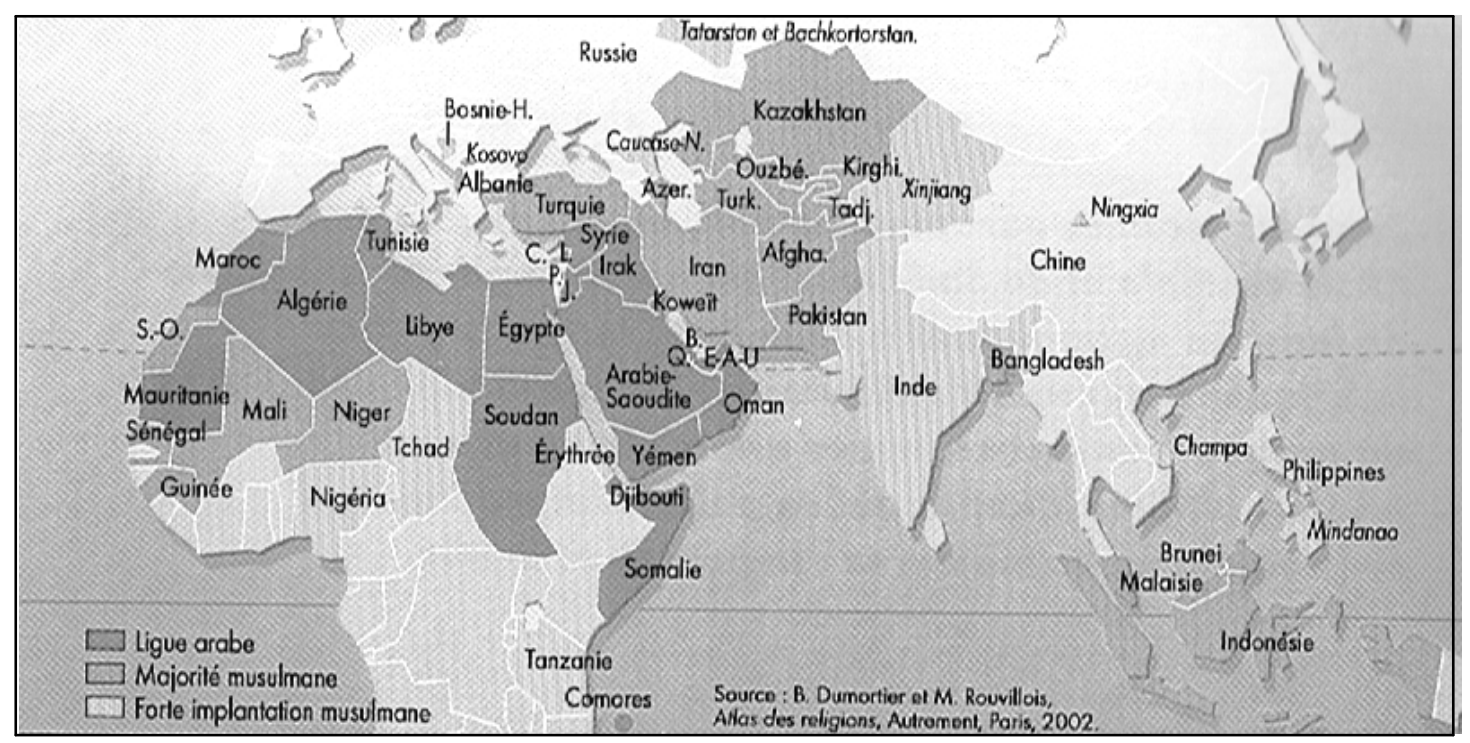

Karte 2. Die Ausbreitung des Arabischen (nach Breton 2003:50)

Auf internationaler politischer Ebene werden über die Arabophonie die Staaten der Großregion Nordafrika in ein zunächst sprachenbasiertes politisches Netzwerk eingebunden, das sich als globaler politischer player verstehen möchte: die Arabische Liga.

Stratum 3 spiegelt die jüngere Kolonialgeschichte des 19. und 20. Jahrhunderts und deren postkoloniale Fortsetzung unter dem Signum der „Globalisierung“ im späten 20. und frühen 21. Jahrhundert, die dem Französischen eine politisch und kulturell dominante Rolle in weiten Teilen Nordafrikas zuwachsen lässt. (Die eher kurzlebigen italienischen Kolonialaktivitäten in Nord- und Nordostafrika haben deutlich weniger soziolinguistisch relevante Spuren hinterlassen.) Es ist interessant $\mathrm{zu}$ beobachten, dass sich in Nordafrika die Großräume bemerkenswerter frankophoner Sprachpraxis nicht mit der Mitgliedschaft in dem internationalen politischen Netzwerk der offiziellen Frankophonie (OIF: Organisation Internationale de la Francophonie) decken, wie die Karten 3 und 4 zeigen. Während im nördlichen Afrika Marokko, Algerien und Tunesien sowie die Westsahara zur espace francophone mit nennenswertem, wenn auch nicht offiziellem Gebrauch der französischen Sprache gezählt werden, gehören von diesen nur Marokko, Tunesien und die Westsahara der OIF an, dazu allerdings auch Ägypten (vgl. dazu in Europa die Mitgliedschaft von Polen, Rumänien und weiteren Balkanstaaten, die ebenfalls nicht zur espace francophone gezählt werden können). 


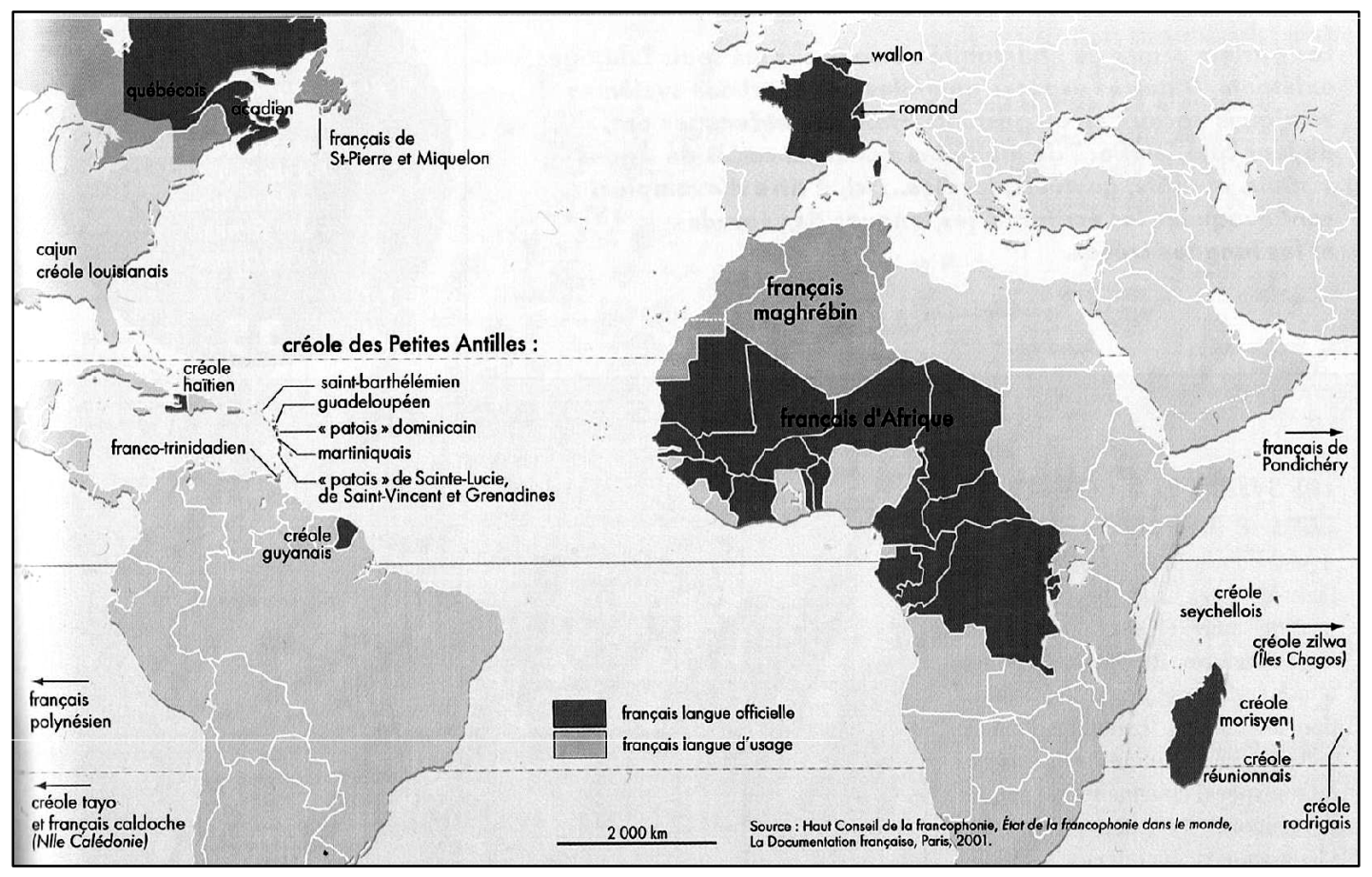

Karte 3. Frankophonie: Staaten mit nennenswertem Gebrauch des Französischen (Espace francophone; Breton 2003:19)

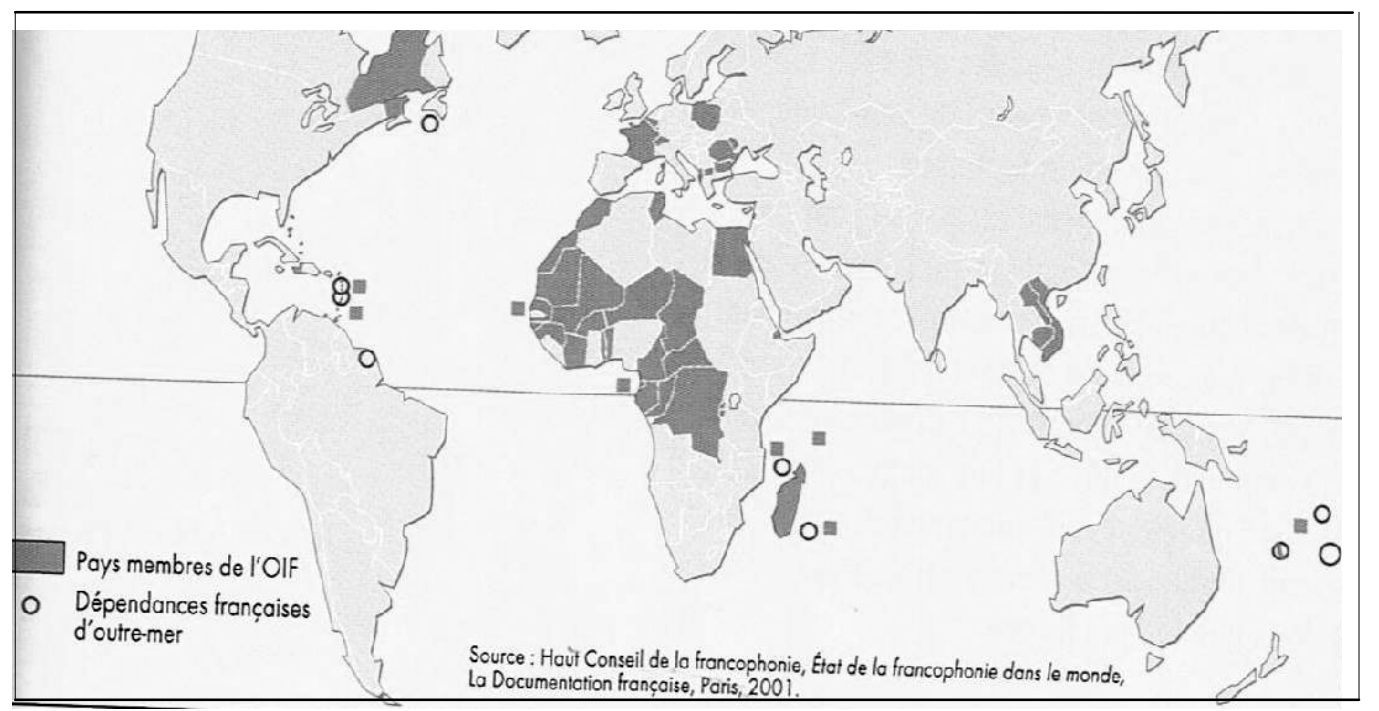

Karte 4. Frankophonie: Mitgliedsstaaten der OIF (Breton 2003:27) 


\section{4. „Polyglossie“ im nördlichen Afrika}

Es gibt in der Soziolinguistik den seit langem etablierten Begriff der „Diglossie“, der ursprünglich ein hierarchisches Macht- und Prestigeverhältnis zwischen zwei Varietäten derselben Sprache beschreibt, von denen die eine als wenig prestigeträchtige Mindervarietät (low variety), die andere hingegen als prestigeträchtige Hoch- oder Standardvarietät (high variety) bezeichnet werden kann. Die als Illustration üblicherweise herangezogenen Beispiele liefern gerade das Arabische (Klassisch-Arabisch bzw. Hocharabisch als high variety, die lokalen arabischen Umgangssprachen als low varieties), aber auch z.B. das Schwyzerdütsch der Schweiz im Verhältnis zur hochdeutschen Standardsprache. Dieser soziolinguistisch sehr brauchbare Begriff lässt sich unschwer auch auf bilinguale Gesellschaften übertragen, in denen zwei verschiedene Sprachen in einem vergleichbar ungleichen Macht- und Prestigeverhältnis stehen, wie es z.B. vielfach in Afrika das Verhältnis zwischen einer einheimischen Sprache und der exkolonialen Amtssprache (z.B. Englisch, Französisch, oder Portugiesisch) betrifft. Bilingualismus und Diglossie sind also verschiedene soziolinguistische Kategorien, wiewohl sie sich beide korrekt ins Deutsche als „Zweisprachigkeit“ übersetzen lassen. Systematisch können wir diese für soziolinguistische Typologien wichtigen Begrifflichkeiten mit den distinktiven Merkmalen [ \pm bilingual] und [ \pm diglossisch] wie folgt darstellen (Tabelle 1):

\begin{tabular}{|c|c|c|c|c|c|}
\hline \multicolumn{3}{|c|}{ monolingualer Kontext } & \multicolumn{3}{|c|}{ bilingualer Kontext } \\
\hline \multirow[t]{2}{*}{ Typ A } & \multirow[t]{2}{*}{ L1 } & [-bilingual] & \multirow[t]{2}{*}{ Typ C } & \multirow[t]{2}{*}{$\mathrm{L} 1+\mathrm{L} 2$} & [+bilingual] \\
\hline & & [-diglossisch] & & & [-diglossisch] \\
\hline \multirow[t]{2}{*}{ Typ B } & \multirow[t]{2}{*}{ L1 } & [-bilingual] & \multirow[t]{2}{*}{ Typ D } & \multirow[t]{2}{*}{$\mathrm{L} 1+\mathrm{L} 2$} & [+bilingual] \\
\hline & & [+diglossisch] & & & [+diglossisch] \\
\hline
\end{tabular}

Tabelle 1. Diglossie in mono- und bilingualen Kontexten

Typ A repräsentiert einen Idealtypus von Einsprachigkeit, bei dem alle sprachinternen Varietäten ein etwa gleiches Prestige besitzen (vielleicht anwendbar auf die Länder Bayern und Baden-Württemberg in der Bundesrepublik Deutschland, in der sich lokale Mundarten wie Alemannisch, Bairisch, Schwäbisch weitgehender allgemeiner Akzeptanz erfreuen, neben Varietäten des Standarddeutschen). Im Gegensatz dazu steht Typ B, der ein deutliches Prestigegefälle zwischen z.B. Hochsprache und „Dialekt" repräsentiert (vergleichbar der Situation in Norddeutschland, wo plattdütsche Varietäten eher als Symbol niedrigen sozialen Prestiges gelten). In einer bilingualen Situation kann das diglossische Gefälle die beiden betroffenen Sprachen einbeziehen, wobei zugleich die soziolinguistische Situation bei sich verändernden historischen Bedingungen einem typologischen Wandel unterworfen werden kann. Nehmen wir das in Europa nahe liegende Beispiel Finnlands. Das heutige Finnland war vor der Unabhängigkeit (1917) für 600 Jahre (bis 1809) Teil des schwedischen Reiches. Aus dieser Zeit stammt die Wahrnehmung des Schwedischen als prestigeträchtige Sprache der Reichsgewalt, der städtischen Kultur und Bildung der feudalen und großbürgerlichen schwedischsprachigen Bevölkerungsteile, während das Finnische weithin als Sprache minderen Prestiges der ländlichen und eher bildungsfernen Bevölkerungsteile galt. Diese Wahrnehmung hat sich in Folge der finnischen Nationalromantik des 19. Jahrhunderts und der staatlichen Unabhängigkeit am 6. Dezember 1917 dramatisch zu Gunsten des Finnischen verändert. Beide Sprachen gelten heute als gleichberechtigte Amtssprachen, allerdings mit Finnisch in einer demographisch und territorial eindeutigen Majoritätsposition. Hinsichtlich 
der Erscheinung des soziolinguistischen Wandels belegt das Beispiel Finnlands, dass und wie sich die bilinguale Situation in relativ rezenter Zeit von dem historischen Typus D (mit Schwedisch als der prestigeträchtigen Sprache) in einen „offiziellen“ Typus C verändert hat, in dem - zumindest de jure - beide Sprachen in einem diglossiefreien Verhältnis zu einander stehen. Dessen ungeachtet ist de facto eine Situation eingetreten, in der erneut ein Typ D zu konstatieren ist, in dem sich nun das Finnische der demographischen und territorialen Majorität zur prestigeträchtigen Sprache zu Lasten des Schwedischen als regionale Minoritätensprache entwickelt hat.)

In Afrika haben wir es weniger mit bilingualen, also zweisprachigen, als mit multilingualen, also vielsprachigen Situationen $\mathrm{zu}$ tun, entsprechend komplex gestaltet sich das Binnenverhältnis der beteiligten Sprachen hinsichtlich demographischer und geographischer Distribution, aber eben auch hinsichtlich ubiquitärer Macht- und Prestigegefälle. Daher spreche ich hier, abgeleitet vom etablierten Begriff der Diglossie, von „Polyglossie“. Dieser Polyglossie-Begriff lässt sich nun sehr passend auf die Situation in Nordafrika anwenden, an der verschiedene als diglossisch wahrgenommene Varietäten des Arabischen mit dem postkolonialen Französisch auf der einen und den vielfältigen berberischen Varietäten auf der anderen Seite eine sehr komplexe soziolinguistische Gemengelage bilden. Die gesellschaftspolitischen Auswirkungen sind dramatisch, da hier unmittelbar Fragen des Sprachenrechts, die zugleich solche von Minoritätenrechten sind, angesprochen werden müssen. Es geht also um Sprachenpolitik, die zugleich Minoritätenpolitik ist und sich entsprechend umfassend mit Fragen der politischen und/oder kulturellen Autonomie von Bevölkerungssegmenten auseinander setzen muss. Und wieder gilt dies vor allem für den Bereich der formalen Bildung unter dem Aspekt, wann und wie lange in welchen Sprachen unterrichtet werden soll oder muss, und wann, wenn überhaupt, ein Wechsel in einen zweite oder dritte Unterrichtssprache erfolgen soll.

Das Problem gewinnt eine besondere Schärfe durch das Wirken von sog. Sprachattitïden, d.h. den in den verschiedenen Bevölkerungsgruppen virulenten Vorurteilen und Klischees über jeweils die „anderen“ und deren Sprachen. Diese Vorurteile und Klischees beruhen auf Ignoranz, also mangelnder eigener Bildung, und führen $\mathrm{zu}$ Ressentiments, die auf Diskriminierung und Ausgrenzung hinaus laufen können; in extremen Fällen führen sie zu Verboten (etwa des Gebrauchs der jeweils eigenen Sprache und kultureller Symbole) und zu Zwangsassimilation. In extremen Fällen haben wir es in der Folge mit sog. ethnischen Säuberungen bis hin zu Annihilierung (Völkermord) zu tun. Die einer ausgegrenzten Gruppe von Menschen eigene Sprache oder Sprachform hat in der Geschichte immer wieder dazu geführt, als Identifikationsmerkmal für Diskriminierung oder gar Annihilierung dieser Gruppe herzuhalten. ${ }^{7}$

Wie könnte nun also ein der soziolinguistischen Situation deskriptiv angemessenes Polyglossie-Modell für die Maghrebstaaten aussehen? Vgl. dazu die folgende schematische Darstellung (Tabelle 2). 


\begin{tabular}{|c|c|c|c|}
\hline & $\begin{array}{l}\text { Gesprochene } \\
\text { Varietäten }\end{array}$ & $\begin{array}{l}\text { Geschriebene } \\
\text { Varietäten }\end{array}$ & $\begin{array}{l}\text { Charakteristische } \\
\text { funktionale Domainen }\end{array}$ \\
\hline \multirow[t]{5}{*}{ HIGH } & & $\begin{array}{l}\text { Klassisch- } \\
\text { ARABISCH }\end{array}$ & Islamische Religion \& Zivilisation \\
\hline & \multicolumn{2}{|l|}{ FRANZÖSISCH } & $\begin{array}{l}\text { Schule, amtliche Diskurse, Politik, } \\
\text { Frz.-sprachige Medien }\end{array}$ \\
\hline & \multicolumn{2}{|c|}{ Modernes Hoch-ARABISCH } & $\begin{array}{l}\text { Kulturelle Diskurse, moderne Prosa, } \\
\text { arabischsprachige Medien }\end{array}$ \\
\hline & $\begin{array}{l}\text { Kairo-ARABISCH } \\
\text { (koine) }\end{array}$ & & $\begin{array}{l}\text { moderne } \\
\text { (Film, TV, Pop-Musik, etc.) }\end{array}$ \\
\hline & $\begin{array}{l}\text { Kolloquial-ARABISCH } \\
\text { ARABISCHe } \\
\text { Dialekte }\end{array}$ & & $\begin{array}{lr}\text { Alltägliches } & \text { Leben } \\
\text { (L1/Muttersprachler } & \text { und } \\
\text { [berberophone] Zweitsprachler) } & \end{array}$ \\
\hline LOW & $\begin{array}{l}\text { BERBER (Rif, Kabyl, } \\
\text { Tashelhit, Tamazight, } \\
\text { Nefusi, Siwi, etc.) }\end{array}$ & & $\begin{array}{l}\text { Familie, } \quad \text { intime } \\
\text { (nur Berber L1/Muttersprachler) }\end{array}$ \\
\hline
\end{tabular}

Tabelle 2. Polyglossie-Modell der Maghrebstaaten

Dieses stark schematisierte Polyglossie-Modell lässt unschwer erkennen, welche Sprachgebrauchsmuster sozial konfliktträchtig sind, interessanterweise sowohl innerhalb des Arabischen im Verhältnis Klassisch-Arabisch/Hocharabisch zu den Kolloquial-Arabischen „Dialekten“, die heute im maghrebinischen Sprachgebrauch quasi nationalisiert z.B. als „Marokkanisch“, „Algerisch“, „Tunesisch“, „Ägyptisch“ bezeichnet werden, als auch im Besonderen zwischen erstsprachigen arabophonen und berberophonen Bevölkerungsteilen. In dieser vom komplexen bilingualen Gefälle zwischen Arabisch und Berberisch charakterisierten und konfliktgeladenen Gemengelage kommt dem Französischen eine weitere und ebenfalls nicht konfliktfreie Rolle zu, die zum einen aus der noch nicht endgültig aufgearbeiteten kolonialen Geschichte rührt, zum anderen für viele zumal jüngere Nordafrikaner den Zugang zu westlich-europäischer Kultur, international kompatibler Hochschulbildung und Teilnahme an der sog. Globalisierung des 21. Jahrhunderts bedeutet.

\section{Ausblick: Konfliktvermeidung durch additiven Multilingualismus?}

Wenn wir ein aus soziolinguistischer Sicht praktikables Modell einer weitgehend konfliktfreien multilingualen Sprachen- und Bildungspolitik entwerfen wollen, dann muss dies auf dem Konzept des „empowerment“, d.h. der gleichberechtigten Repräsentanz und soziokulturellen Wertigkeit aller Erst- bzw. Muttersprachen beruhen. Dazu ist von den erstsprachlichen Gegebenheiten und sprachenpolitischen Zielvorstellungen auszugehen, wie sie das folgende Modell schematisch beschreibt (Tabelle 3). ${ }^{8}$ 


\begin{tabular}{|c|c|c|c|}
\hline \multirow{3}{*}{$\begin{array}{l}\text { L1 } \\
\text { Erstsprache }\end{array}$} & ARABISCH & BERBER & FRANZÖSISCH \\
\hline & Erworbene Diglossie & Neue Diglossie & Traditionelle Diglossie \\
\hline & $\begin{array}{l}\text { 1. L1 Kolloquialarabisch } \\
\text { 2. Klassisch-Arabisch \& } \\
\text { Modernes Hocharabisch } \\
\text { (formale Bildung) }\end{array}$ & 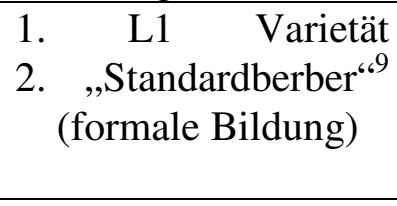 & $\begin{array}{l}\text { 1. L1 regionale Varietät } \\
\text { 2. Standardfranzösisch } \\
\text { (formale Bildung) }\end{array}$ \\
\hline \multirow[b]{2}{*}{$\begin{array}{l}\text { L2 } \\
\text { Zweitsprache }\end{array}$} & & Erworbene Diglossie & Erworbene Diglossie \\
\hline & & $\begin{array}{l}\text { 1. Kolloquialarabisch } \\
\text { 2. Klass. Arabisch \& } \\
\text { Mod. Hocharabisch } \\
\text { (formale Bildung) }\end{array}$ & $\begin{array}{l}\text { 1. Kolloquialarabisch } \\
\text { 2. Klass. Arabisch \& } \\
\text { Mod. Hocharabisch } \\
\text { (formale Bildung) }\end{array}$ \\
\hline $\begin{array}{l}\text { L3 } \\
\text { Fremdsprache }\end{array}$ & $\begin{array}{l}\text { Standardfranzösisch } \\
\text { (formale Bildung) }\end{array}$ & $\begin{array}{l}\text { Standardfranzösisch } \\
\text { (formale Bildung) }\end{array}$ & \\
\hline
\end{tabular}

Tabelle 3. Bi-/trilinguale und bi-/triglossische Situation in den Maghrebstaaten

Die Umsetzung dieses Modells erfordert die Strategie des additiven Multilingualismus im formalen Bildungssystem, ausgehend von einer kontinuierlichen Nutzung der jeweiligen Erstbzw. Muttersprache (Arabisch, Berberisch, Französisch - und zwar jeweils derjenigen NonStandard-Varietät, die die Kinder bei Schuleintritt als L1 beherrschen, ggf. mit Ausnahme des Französischen) als Unterrichtssprachen mindestens bis zum Abschluss des Primarzyklus. ${ }^{10}$ Ein - idealtypisches - additives L1-basiertes bi- bzw. trilinguales Modell könnte wie folgt aussehen (Tabelle 4), in dem die L2 (d.h. die zunächst als Unterrichtsfach eingeführte Zweitsprache) fortschreitend auch als Unterrichtsmedium (im Sinne eines dual media Konzepts) eingesetzt wird, jedoch nicht über ein Maximum von 50\% allen Unterrichts hinaus. Im Falle des Berberischen als L1 bieten sich zwei Optionen an: (a) es wird verzichtet, ein „Standardberber“ zu entwickeln und einzusetzen, in diesem Falle tritt Arabisch als L2 ein, (b) bei Einführung eines „Standardberber“ wäre an ein additiv trilinguales Modell zu denken, mit allerdings bislang kaum erforschter Wirkung bzgl. der erwünschten Entwicklung einer robusten dreisprachigen Kompetenz. 


\begin{tabular}{|c|c|c|}
\hline \multicolumn{3}{|l|}{ Primarzyklus } \\
\hline $\begin{array}{lr}\text { Für } & \text { Schüler } \\
\text { L1/,,Muttersprache““ }\end{array}$ & $\begin{array}{l}\text { Unterrichtsmedium } \\
\text { additiv bi-/trilingual }\end{array}$ & Unterrichtsfächer \\
\hline L1 = Kolloquialarabisch & $\begin{array}{l}L 1(100 \%>50 \%) \\
\& L 2(0 \%>50 \%)\end{array}$ & $\begin{array}{lr}\text { Kolloquialarabisch } & \text { (L1) } \\
\text { Modernes Hocharabisch } & \text { \& } \\
\text { Klassisch-Arabisch } & \text { (L2) } \\
\text { Standardfranzösisch (L3) } & \\
\end{array}$ \\
\hline \multirow[t]{2}{*}{ L1 $=($ Kolloquial- $)$ Berberisch } & 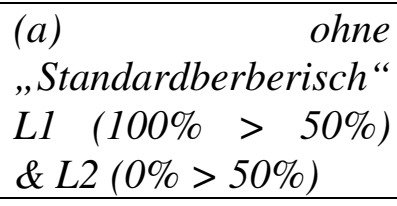 & $\begin{array}{lr}\text { (Kolloquial-)Berberisch } & \text { (L1) } \\
\text { Modernes Hocharabisch } & \text { \& } \\
\text { Klassisch-Arabisch } & \text { (L2) } \\
\text { Standardfranzösisch (L3) } & \\
\end{array}$ \\
\hline & $\begin{array}{l}\text { (b) } \quad \text { mit } \\
\text { "Standardberberisch“ } \\
\text { L1 }(100 \%>50 \%) \\
\& \text { \& } 2 a(0 \%>25 \%) \\
\& L 2 b(0 \%>25 \%)\end{array}$ & $\begin{array}{l}\text { (Kolloquial-)Berberisch (L1) } \\
\text { „Standardberberisch“ (L2b) } \\
\text { Modernes Hocharabisch \& } \\
\text { Klassisch-Arabisch } \\
\text { Standardfranzösisch (L3) }\end{array}$ \\
\hline$L 1=$ Französisch & $\begin{array}{l}L 1(100 \%>50 \%) \\
\& L 2(0 \%>50 \%)\end{array}$ & $\begin{array}{lr}\text { Standardfranzösisch } & \text { (L1) } \\
\text { Modernes Hocharabisch } & \& \\
\text { Klassisch-Arabisch (L2) } & \\
\end{array}$ \\
\hline
\end{tabular}

Tabelle 4. Idealtypischer Primarzyklus: Additiver L1-basierter Multilingualismus

Linguistisches Bildungsziel spätestens zum Ende des Sekundarzyklus wäre eine umfassende bilinguale Kompetenz für L1-Sprecher des Kolloquialarabischen und des Französischen sowie eine trilinguale Kompetenz für L1-Sprecher des Berberischen in den jeweiligen Standardvarietäten (Tabelle 5). Bei begründeter wenn auch optimistischer Annahme, dass nach einem entsprechend erfolgreichen Primarzyklus die L2-Kompetenz der Schüler einen $100 \%$ Einsatz von nicht-L1-Sprachen als Unterrichtsmedium als Basis für Wissenserwerb und kognitive Transferleistungen erlaubt, wäre folgendes idealistische Modell denkbar; im Sinne eines umfassenden Ausbaus der L1 ist diese als Unterrichtsfach bis zum Ende des Sekundarzyklus zu erhalten: 


\begin{tabular}{|c|c|c|}
\hline \multicolumn{3}{|l|}{ Sekundarzyklus } \\
\hline $\begin{array}{lcc}\text { Für } & \text { Schüler } & \text { mit } \\
\text { L1/,,Muttersprache“ } & \end{array}$ & $\begin{array}{l}\text { Unterrichtsmedium } \\
\text { bilingual (dual media) }\end{array}$ & Unterrichtsfächer \\
\hline $\begin{array}{l}\text { L1 } \\
\text { Kolloquialarabisch }\end{array}$ & $\begin{array}{l}\text { L2 Modernes Hocharabisch } \\
\& \\
\text { L3 Standardfranzösisch }\end{array}$ & $\begin{array}{l}\text { Kolloquialarabisch (L1) } \\
\text { Modernes Hocharabisch \& } \\
\text { Klassisch-Arabisch (L2) } \\
\text { Standardfranzösisch (L3) }\end{array}$ \\
\hline $\begin{array}{l}\text { L1 } \\
\text { (Kolloquial-)Berberisch }\end{array}$ & $\begin{array}{l}\text { L2b „Standardberberisch“ } \\
\& \\
\text { L2b Modernes Hocharabisch } \\
\& \text { Klassisch Arabisch }\end{array}$ & $\begin{array}{l}\text { (Kolloquial-)Berberisch (L1) } \\
\text { „Standardberberisch“ (L2b) } \\
\text { Modernes Hocharabisch \& } \\
\text { Klassisch-Arabisch (L2a) } \\
\text { Standardfranzösisch (L3) }\end{array}$ \\
\hline $\begin{array}{l}\text { L1 } \\
\text { Französisch }\end{array}$ & $\begin{array}{l}\text { L1 Standardfranzösisch } \\
\& \\
\text { L2 Modernes Hocharabisch \& } \\
\text { Klassisch Arabisch }\end{array}$ & $\begin{array}{l}\text { Französisch (L1) } \\
\text { Modernes Hocharabisch \& } \\
\text { Klassisch-Arabisch (L2) }\end{array}$ \\
\hline
\end{tabular}

Tabelle 5. Idealtypus Sekundarzyklus: Bi-/Trilingualismus in Standardvarietäten

Sprachenpolitisch und sprachenrechtlich wäre dies durch eine verfassungsmäßig garantierte offizielle Dreisprachigkeit Arabisch-Berberisch-Französisch zu untermauern. ${ }^{12}$

Die praktisch-linguistischen Voraussetzungen für bi- oder trilinguale Lösungsstrategien liegen im Wesentlichen vor, mit Ausnahme der historisch erklärbaren unbefriedigenden Situation für die Berbersprachen: Hier fehlt es weitgehend noch an adäquaten Voraussetzungen für eine praktikable Standardisierung, weil dazu in der Vergangenheit sowohl der politische Wille als auch die nötigen Ressourcen fehlten. Als erschwerend für eine Standardisierung des Berberischen auf supranationaler Ebene, aber bereits auch auf nationaler oder regionaler Ebene, erweist sich dessen linguistische Aufsplitterung. Es muss befürchtet werden, dass ein pan-dialektaler Standard in Lexik und Grammatik (Phonologie, Morphologie, Syntax) soweit von der vertrauten jeweiligen lokalen Varietät eines Lernenden entfernt ist, dass das Erlernen des Standards dem Erlernen einer Fremdsprache vergleichbar wird mit der weiteren Erschwernis, dass dieser Standard tatsächlich von niemandem gesprochen wird und gänzlich auf schriftliche Register beschränkt wäre. ${ }^{13}$ Wie es scheint, hat im Maghreb aber bereits ein politischer Umdenkungsprozess begonnen. Zudem liegt hier ein reiches Potenzial für eine grenzüberschreitende regionale kulturelle Kooperation, indem Ressourcen gebündelt und in der Tat ggf. eine grenzüberschreitende international harmonisierte Standardisierung des Berberischen ins Auge gefasst wird. Aber auch ohne eine Internationalisierung des Projekts spricht aus soziolinguistischer Sicht nichts dagegen, sich umgehend bzw. verstärkt einer Standardisierung etwa eines pan-dialektalen „Marokkanisch-Berberischen“, „AlgerischBerberischen" etc. zuzuwenden. Dabei wird auch die Frage zu beantworten sein, ob und in welchem Ausmaß die anvisierte Trilingualität der Berbersprachigen sich zugleich auch in einem offiziellen Trigraphismus manifestieren soll, denn für das Berberische (ggf. pandialektal übergreifend als Tamazight apostrophiert) stünde ein eigenes Schriftsystem zur Verfügung, das historische Bezüge zu den Altlibyschen Inschriften ausweist und bis heute bei den Tuareg in der südlichen Sahara in Gebrauch ist, nämlich das Tifinagh (Tabelle 6). Allem Anschein nach hat die Verwendung des Tifinagh einen hohen historisch, ideologisch und 
emotional aufgeladenen Symbolwert für die sprachliche, historische und kulturelle Identität derer, die für sich die Eigenbezeichnung Amazigh reklamieren, und hat insbesondere in der berberophonen außerafrikanischen Diaspora eine bemerkenswerte Akzeptanz als Vehikel und Symbol einer autonomen Amazigh-Identität gewonnen.

\begin{tabular}{|l|l|}
\hline Sprache & Schriftsystem \\
\hline $\begin{array}{l}\text { Arabisch Klassisch-, } \\
\text { (Kolloquial-, ARABISCH } \\
\text { Modernes Hocharabisch) }\end{array}$ & \\
\hline Berberisch (= „Tamazight“?) & TIFINAGH \\
\hline Französisch & LATEINISCH \\
\hline
\end{tabular}

Tabelle 6. Nordafrikanischer Trigraphismus

Basis aller soziolinguistisch befriedigenden Lösungen für Nordafrika sind zunächst jedoch

(a) die Akzeptanz einer fundamentalen „offiziellen“ Dreisprachigkeit mit jeweils starker dialektaler Varianz: Arabisch, Berberisch, Französisch,

(b) die Akzeptanz kolloquialer Varietäten in zumindest zwei Sprachen (Arabisch, Berberisch) als bevorzugt geeignete Unterrichtssprachen zunächst im Primarzyklus, basierend auf

(c) der Akzeptanz des pädagogisch-didaktischen Grundprinzips, dass erfolgreiches Lernen vom Bekannten zum Unbekannten fortschreiten muss, d.h. in linguistischer Hinsicht, dass die mit muttersprachlicher Kompetenz beherrschten Kolloquialvarietäten eines jeden Kindes/Schülers Ausgangspunkte für den Erwerb von Standardvarietäten der relevanten Zweit- und Fremdsprachen sein müssen.

Einer besonderen sprachplanerischen Zuwendung bedürfen sowohl das Arabische, dessen Kolloquialvarietäten zunächst offizieller Status als eigene L1 größter Bevölkerungsteile einzuräumen wäre (gern unter nationalisierten Bezeichnungen wie Ägyptisch, Algerisch, Libysch, Marokkanisch, Tunesisch) als auch das Berberische (ggf. unter der pandialektalen Oberbezeichnung Tamazight), für das ggf. regionale (nationale?) Standardvarietäten entwickelt werden müssen. Dabei dürfte nicht verwundern, wenn die drei Sprechergruppen schon aus Gründen der optischen Gleichberechtigung - auf einer die Großregion Nordafrika umfassenden Trigraphie (Arabisch, Lateinisch, Tifinagh) bestünden.

\section{Anmerkungen}

1. Dieser Beitrag geht auf einen Einführungsvortrag zurück, den der Autor im Oktober 2008 in Oran, Algerien, anlässlich des 2. Internationalen Experten-Workshops für Alumni im Rahmen des Deutsch-afrikanischen Alumni-Netzwerkes „GANAA“ zur Thematik von Multilingualismus und Sprachenpolitik in Afrika (und Europa) gehalten hat. Der Autor dankt Claus Altmayer für wertvolle Fragen zur Präzisierung nach der Lektüre einer früheren Version dieses Artikels.

2. So ist denn auch nicht erstaunlich, dass eine der wissenschaftlichen Keimzellen der deutschsprachigen Afrikanistik in der Wiener Ägyptologie zu suchen ist (vgl. Wolff 1981a) und die berberologische Tradition dort bis in das Wirken von H. G. Mukarovsky (1922-1992) erkennbar bleibt. Eben so wenig kann vor diesem Hintergrund verwundern, dass der studierte Austronesist und Afrikanist Alfred Willms 1970 an der Universität Hamburg mit einer Arbeit über die südlichen Berberdialekte in Marokko (Willms 1972) habilitiert wurde am dortigen Institut für Afrikanische 
Sprachen und Kulturen und eine langjährige berberologische Lehrtätigkeit begründete. Die quasi verwaiste europäische Berberologie erfährt in jüngster Vergangenheit eine von deutschen Afrikanisten ins Leben gerufene Renaissance durch eine Konferenzserie („Bayreuth-Frankfurt-Leidener Kolloquium zur Berberologie“). Der Autor selbst betreut zum Zeitpunkt des Schreibens dieses Beitrages am Institut für Afrikanistik an der Universität Leipzig eine afrikanistische Dissertation zur Phonetik und Phonologie des Siwi, der Berbersprache in der Oase Siwa im nordwestlichen Ägypten (Naumann, in Vorb.).

3. Die Nichtwahrnehmung bzw. Negierung dieses Problems mag damit zusammenhängen, dass es im sozial- und wirtschaftswissenschaftlich dominierten mainstream Entwicklungsdiskurs generell als irrelevant oder gar kontraproduktiv gilt, sich seriös mit Fragen von Multilingualismus unter Einbeziehung indigener Sprachen in Afrika auseinanderzusetzen (vgl. dazu Wolff 2006, 2007a, 2007b).

4. Dies gilt vor allem für extreme Problemfälle wie z.B. Kamerun oder Côte d'Ivoire, bei denen sich keine wie auch immer geartete endoglossische oder kombinierte endo- und exoglossische Lösung im Sinne einer offiziellen Sprachenpolitik anzubieten scheint.

5. Als Ägyptisch wird hier eine der Sprachfamilien des Afroasiatischen Sprachstammes bezeichnet; es handelt sich also nicht um die von den Sprechern selbst als „Ägyptisch“ bezeichnete Varietät des gesprochenen Arabisch, wie es heute in Ägypten beheimatet ist.

6. Die Karte zeigt eine dreifache Ausbreitungsintensität: (a) dunkel getönt die Mitglieder der Arabischen Liga (in Afrika: Mauretanien, Marokko, Algerien, Tunesien, Libyen, Ägypten, Sudan, Djibuti); (b) in mittlerer Graustufe Staaten mit muslimischer Mehrheit (in Afrika: Senegal, Guinea, Westsahara, Mali, Niger, Eritrea, Komoren); (c) in heller Graustufe Staaten mit starkem muslimischen Bevölkerungsanteil (in Afrika: Nigeria, Tschad, Tansania).

7. Eines der ältesten Beispiele überliefert die Bibel (Richter 12,5-6): Da die Ephraimiter offenbar keine palatalen Konsonanten in ihrer Sprache kannten, nutzten die ihnen feindlich gesonnenen Gileaditer dieses linguistische Faktum dazu, Flüchtlinge aus Ephraim zu identifizieren und massenhaft zu töten, indem sie sie zwangen, das hebräische Wort Schibbolet (,Ähre; Wasserflut") auszusprechen. Wenn die Betroffenen das Wort mit einem alveolaren Anlautkonsonanten (also Sibbolet) aussprachen statt mit einem palatalen Anlautkonsonanten (wie in Schibbolet), erschlugen die Gileaditer sie ,,an den Furten des Jordans, so dass zu der Zeit von Ephraim fielen zweiundvierzigtausend“, wie es in der Bibel heißt. Vergleichbare Fälle wurden aus dem nigerianischen Bürgerkrieg in den späten 1960er Jahren berichtet bzgl. der Identifizierung Igbo-sprachiger Flüchtlinge durch Bundestruppen.

8. Modernes Hocharabisch und Klassisch-Arabisch werden im Folgenden als L2 (bzw. L2a) zusammengefasst, da beide im formalen Bildungssystem als Zweitsprache vermittelt werden. Im allgemeinen Sprachgebrauch im Maghreb entspricht dies dem Gegensatz von „Arabisch“ (= Modernes Hocharabisch/Klassisch-Arabisch) und z.B. „Algerisch“, „Tunesisch“ etc. als nationalisierten Bezeichnungen für regionales Kolloquialarabisch.

9. Zur besonderen Problematik bei der Entwicklung eines „Standardberber“ s. weiter unten.

10. Im Falle des Französischen als L1 könnte auf die Nutzung regionaler Varietäten statt Standardfranzösisch verzichtet werden, da der Einsatz des Standardfranzösischen von Schulbeginn an, nach dem Modell des Mutterlandes Frankreich, als pädagogisch 
machbar gelten kann. Angesichts der Minderheit, für die L1 Französisch anzunehmen ist, dürfte die „politisch inkorrekte“ Tatsache kaum ins Gewicht fallen, dass das vorgeschlagene System für L1-Sprecher des Französischen „nur“ Zweisprachigkeit statt Dreisprachigkeit vorsieht. Im Falle von L1-Sprechern des Kolloquialarabischen liegt, trotz der übergreifenden Bezeichnung „Arabisch“, hinsichtlich Kolloquialarabisch und Modernem Hocharabisch/Klassisch-Arabisch tatsächliche Zweisprachigkeit vor, in Verbindung mit Französisch also Dreisprachigkeit. In diesem Sinne kommt das System bei den L1-Berbersprachigen zu einem viersprachigen Ziel dies allerdings nur unter der Voraussetzung, dass die empowerment Strategie für das Berberische auf der - durchaus problematischen - Einführung eines „Standardberber“ besteht.

11. Die angegebenen Prozentzahlen beziehen sich auf die sukzessive Anpassung der additiven Nutzung von Unterrichtssprachen: In dem Maße, wie die zunächst ausschließlich (100\%) eingesetzte L1 zugunsten der Einführung einer L2 jeweils auf mindestens 50\% reduziert wird, kann die L2 auf maximal 50\% ausgedehnt werden. Der hier als optimal gesetzte Schwellenwert von $50 \%$ bezieht sich auf Ergebnisse vergleichender Untersuchungen $\mathrm{zu}$ bilingualen Bildungsstrategien in Afrika (vgl. Alidou et al. 2006).

12. Soziolinguistisches Faktum bliebe jedoch, dass wir es bei dieser „offiziellen Dreisprachigkeit" mit einer faktischen Fünfsprachigkeit zu tun hätten: (1) nationales Kolloquialarabisch, (2) Hoch-/Klassisch-Arabisch, (3) Kolloquialberberisch, (4) „Standardberber“, (5) Standardfranzösisch.

13. Dies wiederum macht die Situation des Berberischen der bekannten „diglossalen“ Situation des Arabischen ähnlich!

\section{Bibliographie}

Alidou, H., A. Boly, B. Brock-Utne, Y.S. Diallo, K. Heugh, and H.E. Wolff (Hgg.) 2006 (in press). Optimizing learning and education in Africa - the language factor: a stocktaking research on mother tongue and bilingual education in Sub-Saharan Africa. Hamburg: UNESCO Institute of Lifelong Learning.

Breton, R. 2003. Atlas des languages du monde. Une pluralité fragile. Paris: Éditions Autrement.

Hayward, R. J. 2000. Afroasiatic. In B. Heine und D. Nurse (Hgg.) African Languages. An Introduction. Cambridge: Cambridge University Press. pp. 74-98

Heine, B. and D. Nurse (Hgg.) 2000. African Languages. An Introduction. Cambridge: Cambridge University Press.

Naumann, Ch. (In Vorbereitung.). An Acoustically-based Phonology and Morphophonology of Siwi (Berber).

Willms, A. 1972. Grammatik der südlichen Beraberdialekte. (Afrikanistische Forschungen 6.) Glückstadt und Hamburg: J.J. Augustin.

Whiteley, W.H. 1974. Language in Kenya. Nairobi: Oxford University Press.

Wolff, H.E. 2007a. Die afrikanischen Sprachen im 21. Jahrhundert: Herausforderungen and Politik und Wissenschaft. In Jahrbuch für Europäische Überseegeschichte 7. Wiesbaden: Harrassowitz. pp. 189-219.

Wolff, H.E. 2007b. Was sie sagen und was sie tun: Die „Sprachenfrage in Afrika” im politischen Diskurs. In B. Streck (Hg.) Die gezeigte und die verborgene Kultur. Wiesbaden: Harrassowitz. pp. 103-124. 
Wolff, H.E. 2006. The language factor in discourse on development and education in Africa. In Kembo-Sure, S. Mwangi und N.O. Ogechi (Hgg.) Language Planning for Development in Africa. Eldoret: Moi University Press. pp. 1-22.

Wolff, H.E. 1981a. Die Erforschung der afrikanischen Sprachen: Geschichte und Konzeptionen. In B. Heine, Th.C. Schadeberg und E. Wolff (Hgg.) Die Sprachen Afrikas. Hamburg: Helmut Buske. pp. 17-43.

Wolff, H.E. 1981b. Die Berbersprachen. In B. Heine, Th.C. Schadeberg und E. Wolff (Hgg.) Die Sprachen Afrikas. Hamburg: Helmut Buske. pp. 17-43.

\section{Biographical note}

H. Ekkehard Wolff, born in 1944, received his Ph.D. in 1972. In 1980 he was habilitated in the field of African languages and culture (African Languages and Linguistics) at the University of Hamburg. From 1994 until 2009 he was chair of African Languages and Linguistics at the University of Leipzig. Besides Hamburg and Leipzig he also taught at anglophone and francophone universities in Western Africa (Nigeria, Niger) for a number of years. Due to expeditions, informational- and educational trips he visited more than 20 countries in Africa. His research interests include descriptive and comparative African linguistics, language contact research, language art, sociolinguistics and applied African linguistics. 